\title{
Envelhecimento Físico de Sistemas DGEBA/DDM Investigado por Análise Térmica (DSC/DMA)
}

\author{
Izabel C. Riegel, Liane L. Freitas e Dimitrios Samios
}

Resumo: Neste trabalho, estudou-se o efeito do envelhecimento físico nas propriedades térmicas e mecânicas em sistemas diglicidil éter do bisfenol-A (DGEBA)/diaminodifenilmetano (DDM), em função do grau de conversão, induzido pela cura e do tempo de envelhecimento. A cura isotérmica foi realizada em uma etapa a $115^{\circ} \mathrm{C}$ por diversos tempos e o envelhecimento foi conduzido a $100^{\circ} \mathrm{C}$ por períodos de 240 a $4320 \mathrm{~min}$. Considerando que o envelhecimento físico acarreta variações estruturais as quais afetam tanto o desempenho mecânico quanto as propriedades termodinâmicas do material, as técnicas de DSC e DMA são complementares. Através de Calorimetria Exploratória Diferencial (DSC), observou-se que o envelhecimento físico está associado ao pico endotérmico que ocorre na região da transição vítrea e que a entalpia de relaxação, calculada a partir da área deste pico, aumenta gradualmente com o tempo de envelhecimento. Os resultados obtidos por Análise Dinâmico-Mecânica (DMA) mostraram um aumento do módulo elástico E' com o tempo de envelhecimento. As velocidades de envelhecimento foram obtidas a partir da temperatura do pico endotérmico, a partir do módulo elástico E' e a partir da temperatura de transição vítrea e resultaram tanto menores quanto maior o grau de conversão da matriz. Os resultados salientam a importância da seleção adequada das condições de cura para que se possam obter as melhores propriedades destes materiais. A importância dos fenômenos observados é considerada, tendo-se em vista a grande utilização e aplicabilidade das resinas epóxi.

Palavras-chave: Resinas epóxi, envelhecimento físico, DSC, DMA

\section{Introdução}

As resinas epóxi são amplamente utilizadas comercialmente devido à diversidade de propriedades que podem apresentar em virtude da seleção adequada dos reagentes e processos de cura. Dentre as características mais interessantes estão: inércia química e biológica, boas propriedades adesivas e elétricas bem como estabilidade estrutural e baixa permeabilidade ${ }^{[1]}$. As propriedades desejáveis destas resinas normalmente aparecem após a cura, sendo que estas podem modificar-se por conseqüência do transporte, armazenamento e utilização destes materiais. As etapas de cura transformam os reagentes epoxidílicos de baixa massa molar em um material altamente reticulado com estrutura tridimensional, a qual envolve segmentos da resina e do agente de cura (ou endurecedor). Uma das aplicações industriais do sistema DGEBA/DDM curado é na encapsulação de transformadores de for- 
ça a seco, uso de interesse deste trabalho. A encapsulação é um método que visa, principalmente, manter altos níveis de isolamento elétrico entre condutores, conecções, componentes e outras partes elétricas bem como estabilidade estrutural.

O sistema curado DGEBA/DDM constitui um polímero amorfo que se encontra em seu estado vítreo à temperatura ambiente. Os materiais amorfos vítreos, reticulados ou não, podem ser concebidos como líquidos superresfriados ${ }^{[2]}$, os quais possuem excesso termodinâmico de volume e entalpia. O processo que levará estes materiais a, espontaneamente, densificarem-se no sentido de atingirem um estado de equilíbrio metaestável (relaxação estrutural) denomina-se envelhecimento físico e, portanto, ocorrerá em todos os materiais vítreos, quer sejam poliméricos, monoméricos, orgânicos ou inorgânicos ${ }^{[3,4]}$. A influência do envelhecimento físico nas propriedades viscoelásticas das resinas epóxi baseadas no DGEBA foi bastante estudada por McKenna e colaboradores, pois esta resina serve como modelo no estudo das estruturas reticuladas $^{[5-9]}$. A relaxação estrutural é conseqüência do estado de não equilíbrio do estado vítreo, e a velocidade de ocorrência deste processo depende da temperatura, da pressão e da história do material vítreo. Todas as propriedades que dependem do volume específico (ou do volume livre) se alteram durante este processo e, portanto, o envelhecimento físico possui importância prática na determinação das propriedades de engenharia destes vidros.

O objetivo deste estudo foi avaliar o efeito do envelhecimento físico nas propriedades térmicas e mecânicas de uma série de sistemas DGEBA/ DDM, submetidos a diferentes processos de cura e envelhecimento, por meio de duas técnicas de análise térmica: DSC e DMA. A partir dos resultados obtidos foi possível calcular a velocidade de envelhecimento para os diferentes sistemas.

\section{Experimental}

Os reagentes utilizados (Figura 1) foram a resina epóxi diglicidil éter do bisfenol-A (DGEBA) e a diamina aromática tetrafuncional, 4,4'diaminodifenilmetano (DDM). Estes foram misturados estequiometricamente $(2: 1)$ e homogeneizados a $90^{\circ} \mathrm{C}$, sob vigorosa agitação. Após este procedimen-

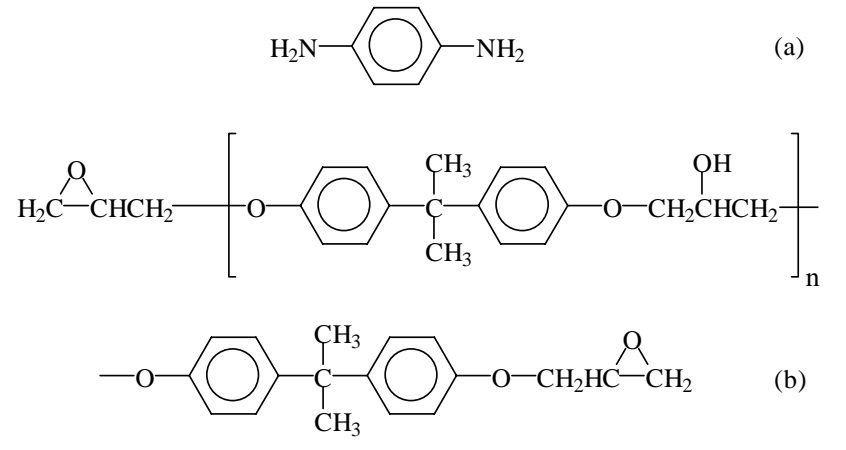

Figura 1. Estrutura química dos reagentes: (a) diaminodifenilmetano (DDM); (b) diglicidil éter do bisfenol-A (DGEBA).

to, que originou um líquido viscoso e transparente, a mistura reacional foi conduzida à cura isotérmica a $115^{\circ} \mathrm{C}$ por diversos tempos (tc). Cada tempo de cura utilizado gera um sistema com determinado grau de conversão ${ }^{[10]}$. Após, cada espécie foi envelhecida a $100^{\circ} \mathrm{C}$ (Ta) por períodos que variaram de 240 a 4320 minutos (ta). A seleção da temperatura de $100^{\circ} \mathrm{C}$ ocorreu após a determinação da temperatura de transição vítrea ( $\mathrm{Tg}$ ) dos sistemas não envelhecidos e deu-se devido a esta ser inferior à temperatura de transição vítrea de todos os sistemas.

Para os experimentos de DSC, a mistura reacional foi curada e envelhecida diretamente em cápsulas apropriadas. Estes foram realizados em um equipamento Perkin-Elmer, modelo DSC-4, com taxa de aquecimento de $\phi=10^{\circ} \mathrm{C} / \mathrm{min}$ em um intervalo de temperatura de 40 a $250^{\circ} \mathrm{C}$.

Para os experimentos de DMA, os reagentes foram homogeneizados diretamente em molde de alumínio ( $8 \times 4 \times 1 \mathrm{~mm}$ ), no qual foram submetidos aos processos de cura e envelhecimento. As medidas de DMA foram realizadas em um analisador dinâmico-mecânico, PL-DMTA, fabricado pela Polymer Laboratories, no modo flexão, nas freqüências de 0,$1 ; 1,0$ e $10 \mathrm{~Hz}$. A taxa de aquecimento foi de $2^{\circ} \mathrm{C} / \mathrm{min}$ em um intervalo de temperatura de $-150^{\circ} \mathrm{C}$ a $200^{\circ} \mathrm{C}$, sob atmosfera de nitrogênio.

\section{Resultados e Discussão}

\section{Evento Endotérmico}

Devido às relaxações estruturais decorrentes do processo de envelhecimento físico, a entalpia do sistema amorfo diminui e, conseqüentemente a variação de entalpia $(\Delta \mathrm{H})$ relacionada com o pro- 
cesso de relaxação aumenta. $\mathrm{O}$ trabalho pioneiro de Ophir ${ }^{[11]}$ e colaboradores relacionou a relaxação de entalpia às mudanças nas propriedades mecânicas de estruturas epóxi, por conseqüência do envelhecimento em temperaturas abaixo da $\mathrm{Tg}$. Este trabalho foi um dos primeiros a atribuir aos processos de relaxação as mudanças nas propriedades das resinas epóxi. Algumas interpretações errôneas, relativas a este fenômeno, ocorreram antes deste trabalho. Esta confusão originou-se devido à presença de inomogeneidades nas matrizes poliméricas reticuladas. Desta forma, Kreibich e Schmid ${ }^{[12]}$ atribuíram o pico endotérmico apresentado pelas amostras envelhecidas à separação de fase entre regiões de alta e baixa densidades de reticulação. A posição e intensidade do pico endotérmico dependem das condições de envelhecimento, isto é, temperatura e tempo. A extensão dos processos de relaxação entálpica em resinas parcialmente curadas depende do grau de conversão e, subseqüentemente, da densidade de reticulação da matriz polimérica. A Figura 2 demonstra esquematicamente como o valor de $\Delta \mathrm{H}$ comporta-se em função da temperatura, de acordo com a história térmica executada neste trabalho. As amostras encapsuladas, já curadas, foram resfriadas até a temperatura de envelhecimento Ta $\left(100^{\circ} \mathrm{C}\right)$ - segmento $\mathrm{AB}$ - mantidas em Ta por um tempo ta- segmento $\mathrm{BC}$ - e depois resfriadas até $\mathrm{T}_{0}\left(40^{\circ} \mathrm{C}\right)$, temperatura inicial do experimento de DSC- segmento CO- dando-se início ao aquecimento a $10^{\circ} \mathrm{C} / \mathrm{min}(\phi)$ até $\operatorname{Tf}\left(250^{\circ} \mathrm{C}\right)$, tempera-

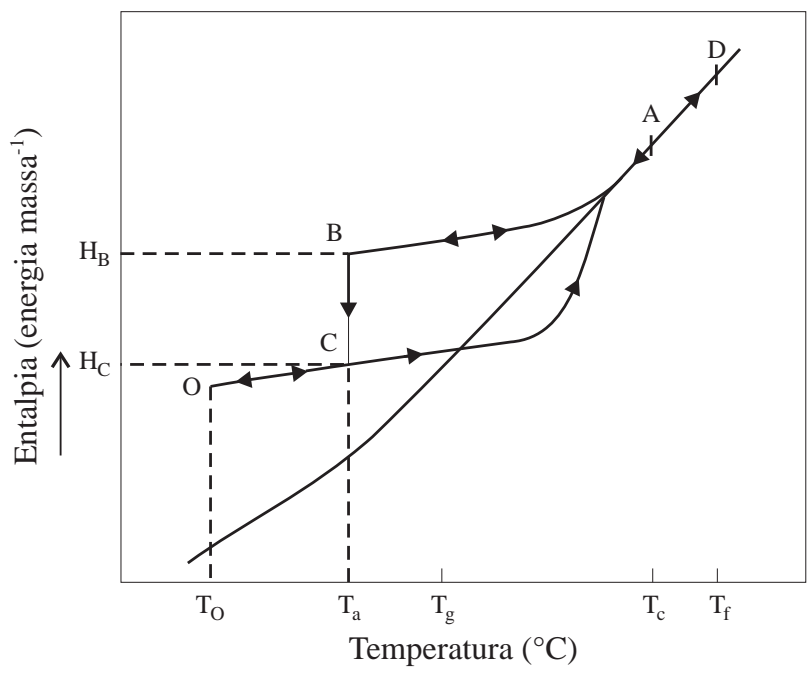

Figura 2. Diagrama esquemático da evolução da entalpia durante uma história térmica incluindo um envelhecimento isotérmico a Ta (caminho ABCOCD). tura final- segmento $\mathrm{OD}$. $\mathrm{O}$ valor de $\Delta \mathrm{H}$ pode ser calculado segundo a equação ${ }^{[13]}$ :

$$
\Delta \mathrm{H}=\mathrm{H}_{\mathrm{B}}(\mathrm{Ta}, \mathrm{to})-\mathrm{H}_{\mathrm{C}}(\mathrm{Ta}, \text { ta })
$$

e também por,

$$
\Delta H=\int_{T 1}^{T o} C_{p}(e n v) d T-\int_{T 1}^{T o} C_{p}(r e f) d T
$$

onde $\mathrm{C}_{\mathrm{p}}$ (env) e $\mathrm{C}_{\mathrm{p}}$ (ref) são as capacidades caloríficas da amostra envelhecida e da referência, respectivamente.

As curvas DSC das amostras curadas a 45 e 90 min estão apresentadas na Figura 3. Observase que a amostra curada durante 45 min (Figura 3a) e não submetida ao envelhecimento ( $\mathrm{ta}=0 \mathrm{~min})$ apresenta a ocorrência de um evento exotérmico que se relaciona à entalpia residual de cura, responsável pela complementação do processo de reticulação. No entanto, após o envelhecimento,

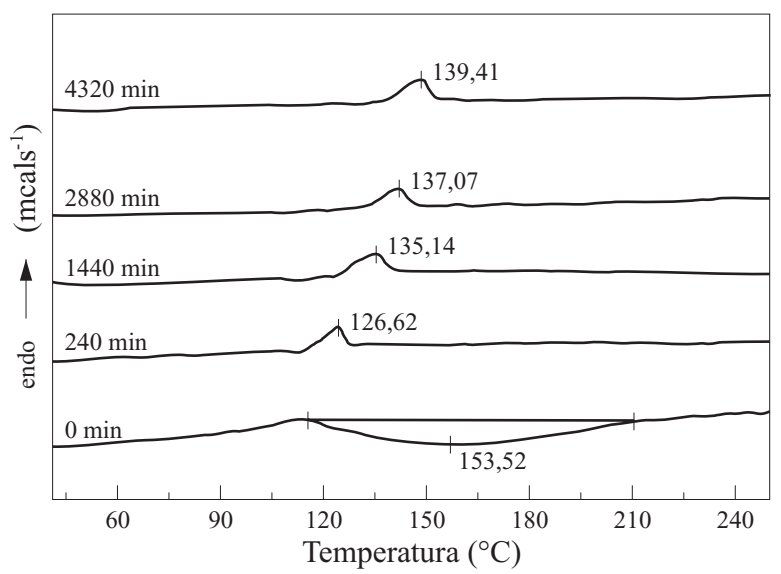

(a)

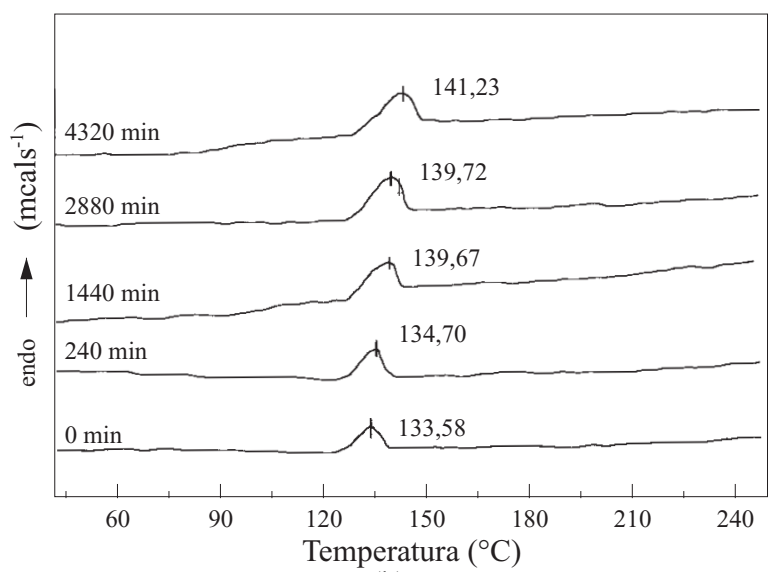

(b)

Figura 3. Curvas DSC para o sistema DGEBA/DDM curado a $115^{\circ} \mathrm{C}$ por (a) $45 \mathrm{~min}$ e (b) $90 \mathrm{~min}$ e envelhecido a $100^{\circ} \mathrm{C}$ durante os tempos indicados. $\phi=10^{\circ} \mathrm{C} / \mathrm{min}$. 
todos os sistemas mostram a ocorrência de um evento endotérmico, que denota a relaxação de entalpia ocorrida. O mesmo comportamento apresentado pelo sistema curado por $45 \mathrm{~min}$ foi encontrado nas amostras curadas por 20 e $30 \mathrm{~min}$. Todos os sistemas curados a 90 min (Figura 3b) e também os curados a $60 \mathrm{~min}$, envelhecidos e não envelhecidos, mostram a ocorrência do pico endotérmico, evidenciando que os tempos de cura de 60 e 90 min levam este sistema reacional à sua completa conversão, na temperatura de cura utilizada $\left(\mathrm{Tc}=115^{\circ} \mathrm{C}\right)$. Imediatamente após os processos de cura e envelhecimento, as temperaturas de transição vítrea destes sistemas foram determinadas por DMA, devido à sobreposição do pico endotérmico à região da transição vítrea.

\section{Entalpia de Relaxação x Tempo de Envelhecimento}

Determinou-se a temperatura em que ocorre o máximo do pico endotérmico $(\mathrm{Tt})$ através das curvas DSC (Figura 3) e calculou-se a área do pico, ou seja, a entalpia de relaxação $\left(\Delta \mathrm{H} / \mathrm{cal}^{-\mathrm{g}^{-1}}\right)$. Os dados são apresentados na Tabela 1 , onde podemos observar o aumento de $\Delta \mathrm{H}$ com o tempo de envelhecimento.

A Figura 4 mostra a evolução da temperatura de ocorrência do máximo do pico endotérmico, Tt, em função do logaritmo do tempo de envelhecimento, log ta. Nesta figura, pode-se observar a relação linear entre $\mathrm{Tt}$ e $\log$ ta. As inclinações das retas, segundo ajuste linear, demonstra claramente que o aumento de $\mathrm{Tt}$ ocorre mais acentuadamente quanto menor o tempo de cura, isto é, o efeito de ta em $\mathrm{Tt}$ é maior nos sistemas curados durante menores tempos. Ainda, o aumento do tempo de envelhecimento causa uma aproximação dos valores de $\mathrm{Tt}$ a qual sugere a tendência do sistema em alcançar seu estado de equilíbrio intrínseco.

Tabela 1. Entalpia de Relaxação $\left(\Delta \mathrm{H} / \mathrm{cal} \mathrm{g}^{-1}\right)$.

\begin{tabular}{ccccc}
\hline $\begin{array}{c}\mathbf{t a}^{(\mathbf{a})}(\mathbf{m i n}) \rightarrow \\
\mathbf{t c}^{(\mathbf{b})}(\mathbf{m i n}) \downarrow\end{array}$ & $\mathbf{2 4 0}$ & $\mathbf{1 4 4 0}$ & $\mathbf{2 8 8 0}$ & $\mathbf{4 3 2 0}$ \\
\hline 20 & 1,6 & 1,7 & 1,7 & 1,8 \\
30 & 1,6 & 1,6 & 1,7 & 1,9 \\
45 & 0,9 & 1,0 & 1,0 & 1,1 \\
60 & 0,9 & 1,1 & 1,1 & 1,2 \\
90 & 1,0 & 1,1 & 1,4 & 1,5 \\
\hline
\end{tabular}

(a) ta é o tempo de envelhecimento a $100^{\circ} \mathrm{C}$;

(b) tc é o tempo de cura a $115^{\circ} \mathrm{C}$.

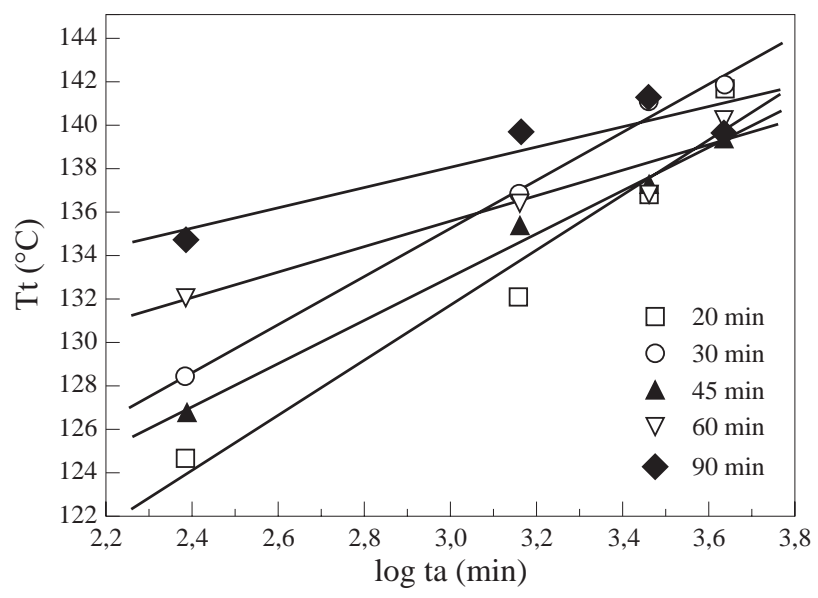

Figura 4. Temperatura do pico endotérmico $(\mathrm{Tt})$ versus logaritmo do tempo de envelhecimento, para diferentes tempos de cura a $115^{\circ} \mathrm{C}$.

O processo de relaxação estrutural origina um decréscimo simultâneo do volume livre e da mobilidade segmental, a medida que o sistema tenta alcançar seu estado de equilíbrio. A mobilidade segmental dos termorrígidos pode refletir os movimentos dos segmentos da cadeia e dos finais de cadeia das regiões reticuladas, podendo também relacionar-se com o movimento de segmentos não totalmente reagidos, sendo que estes movimentos tornam-se mais dificultados com o aumento do grau de conversão da matriz ${ }^{[2,14]}$.

Imediatamente após a primeira varredura de DSC $\left(40^{\circ} \mathrm{C} \rightarrow 250^{\circ} \mathrm{C}\right)$, onde foram determinados os valores de $\Delta \mathrm{H}$ e $\mathrm{Tt}$, as amostras foram mantidas na temperatura final por $10 \mathrm{~min}$, resfriadas rapidamente e, então, foi realizada uma nova corrida nas mesmas condições da anterior. Nesta segunda varredura foi possível determinar o valor da Tg para todas as amostras, não sendo observado nenhum evento térmico quer seja endo ou exotérmico, sendo que os valores encontrados são muito próximos (Tabela 2). Isto demonstra que o processo executado neste trabalho não envolveu degradação ou modificação quí-

Tabela 2. Temperaturas de Transição Vítrea, $\operatorname{Tg}\left({ }^{\circ} \mathrm{C}\right)$, determinadas por DSC, segunda varredura.

\begin{tabular}{cccccc}
\hline $\begin{array}{c}\mathbf{t a}^{(\mathbf{a})}(\mathbf{m i n}) \rightarrow \\
\mathbf{t c}^{(\mathbf{b})}(\mathbf{m i n}) \downarrow\end{array}$ & $\mathbf{0}$ & $\mathbf{2 4 0}$ & $\mathbf{1 4 4 0}$ & $\mathbf{2 8 8 0}$ & $\mathbf{4 3 2 0}$ \\
\hline 20 & 162,0 & 158,6 & 159,5 & 160,8 & 158,2 \\
30 & 159,3 & 164,8 & 162,7 & 162,4 & 161,0 \\
45 & 162,4 & 164,5 & 162,2 & 160,1 & 159,7 \\
60 & 162,3 & 162,9 & 163,4 & 161,0 & 160,5 \\
90 & 161,7 & 162,0 & 163,2 & 162,6 & 158,1 \\
\hline
\end{tabular}

(a) ta é o tempo de envelhecimento a $100^{\circ} \mathrm{C}$;

(b) tc é o tempo de cura a $115^{\circ} \mathrm{C}$. 
mica, somente acomodações estruturais, pois o aquecimento das amostras acima da sua Tg apagou a história térmica, levando todas as amostras a virtualmente o mesmo valor de $\mathrm{Tg}$, independente dos tempos e temperaturas de cura e envelhecimento inicialmente utilizados.

\section{Módulo Dinâmico de Armazenamento}

Neste estudo, foi monitorado o valor da componente real do módulo dinâmico, E'(módulo de armazenamento), a $100^{\circ} \mathrm{C}$ (temperatura de envelhecimento), pois sabe-se que as mudanças esperadas para esta propriedade encontram-se localizadas em torno da $\mathrm{Ta}^{[2,14,15,16]}$ (Figura 5). Esta localização de efeitos deve-se ao fato de cada sistema possuir um espectro de relaxação, sendo que cada mecanismo associado a este espectro relaciona-se a uma certa escala de movimento e a um certo tempo, os quais são dependentes da temperatura em que o sistema se encontra. A uma dada temperatura de envelhecimento físico, cada mecanismo de relaxação está inserido em um dos três grupos seguintes: (1) elementos relacionados a movimentos de larga escala espacial que estão praticamente imobilizados devido às restrições do ambiente e, portanto, não participam do processo de envelhecimento; (2) elementos relacionados a movimentos de média escala espacial que estão impedidos pelas vizinhanças mas, mesmo assim, podem mover-se participando do envelhecimento e (3) elementos relacionados a movimentos de curta ou curtíssima escala, que encontram-se totalmente livres e estão em um estado de equilíbrio local. Elementos do grupo dois rearranjam-se no envelhecimento na tentativa de atingir seu estado de equilíbrio, levando a impedimentos produzidos por seus próprios movimentos, enquanto que os elementos dos grupos um e três, mantêm-se essencialmente inalterados: este constitui o processo de envelhecimento.

$\mathrm{O}$ processo de envelhecimento isotérmico a temperaturas menores que a $\mathrm{Tg}(\mathrm{Ta}<\mathrm{Tg})$ leva ao retardamento de certos movimentos (grupo dois) cuja escala espacial aumenta com a Ta. Este, portanto pode ser considerado como um mecanismo de difusão molecular progressivamente retardante, no qual sua amplitude, que é função da temperatura, é gradativamente imobilizada fisicamente. Este efeito localizado indica, então, que elementos específicos de relaxações intermediárias estão envolvidos no processo de envelhecimento físico a

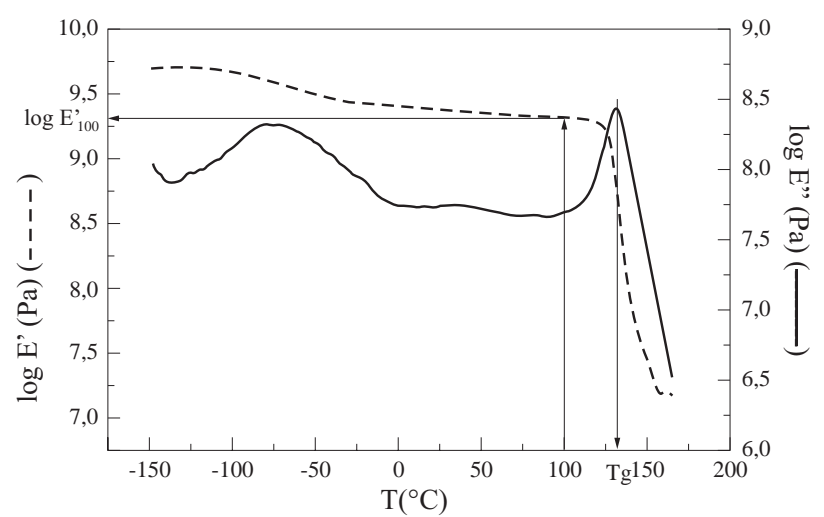

Figura 5. Método de obtenção do módulo de armazenamento E' a $100^{\circ} \mathrm{C}(\mathrm{Ta})$ e da $\mathrm{Tg}$ dos sistemas DGEBA/DDM via DMA. Curva obtida a $1,0 \mathrm{~Hz}$.

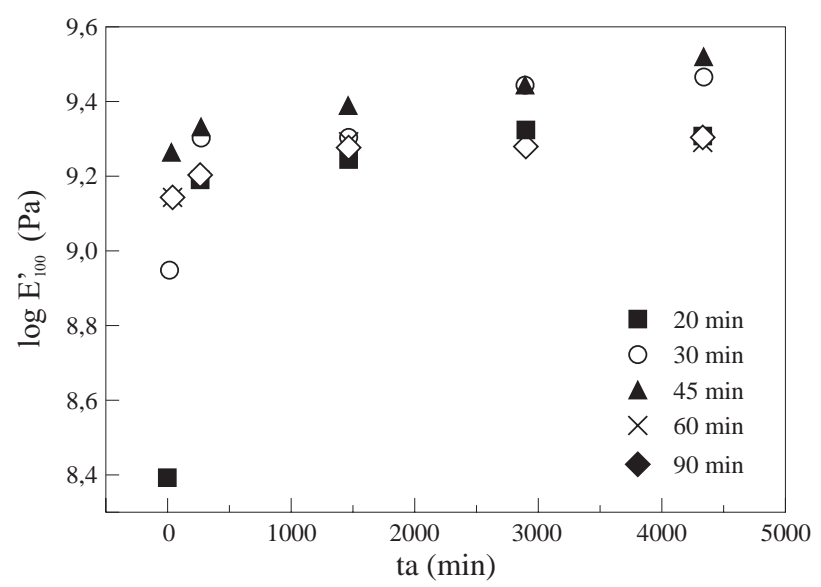

Figura 6. Evolução do módulo de armazenamento $\mathrm{E}^{\prime}$ a $100^{\circ} \mathrm{C}(\mathrm{Ta})$ do sistema DGEBA/DDM com o tempo de envelhecimento. Tempos de cura (tc) indicados.

uma temperatura particular. Esta localização de efeitos no envelhecimento físico sugere que não será necessário aquecer-se a amostra envelhecida até uma temperatura acima da Tg para apagálos. Esta constitui uma característica muito importante do ponto de vista industrial ${ }^{[16]}$.

A Figura 6 mostra os valores de $\log \mathrm{E}^{\prime}$ a $100^{\circ} \mathrm{C}$ (Ta) dos sistemas $\left(\mathrm{E}_{100}{ }_{100}\right.$, obtidos a $1,0 \mathrm{~Hz}$, podendo-se observar uma tendência crescente de E' com o tempo de envelhecimento, evidenciando um aumento da rigidez do material. O aumento do módulo está associado com a redução espontânea do excesso de volume livre. O mesmo comportamento foi encontrado nas medidas realizadas a $0,1 \mathrm{e} 10 \mathrm{~Hz}$.

\section{Velocidade de Envelhecimento}

Neste trabalho, a velocidade de envelhecimento será definida a partir dos valores de $\mathrm{Tt}$ (temperatura do pico endotérmico, via DSC), a partir dos 


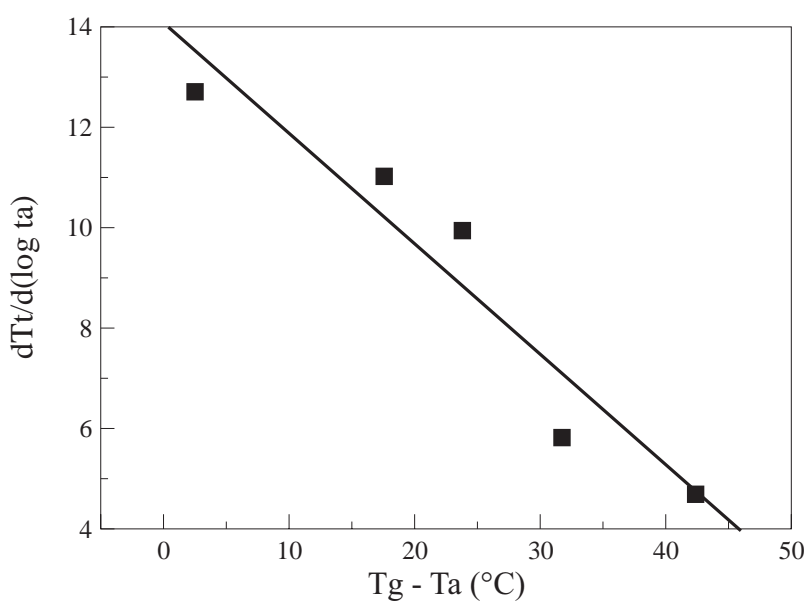

Figura 7. Velocidades de envelhecimento a partir das temperaturas $\mathrm{Tt}$ em função de $(\mathrm{Tg}-\mathrm{Ta})$.

valores do módulo de armazenamento a $100^{\circ} \mathrm{C}$, E' ${ }_{100}$ e a partir dos valores das temperaturas de transição vítrea dos sistemas determinados por DMA pela inflexão da curva do módulo de perda (E”), a 1,0 Hz (Figura 5).

A Figura 7 apresenta o comportamento da velocidade de envelhecimento considerando os valores de $\mathrm{Tt}, \mathrm{Ra}_{\mathrm{Tt}}$, em função de (Tg-Ta), que é uma medida do afastamento do equilíbrio ${ }^{[13,14]}$. $\mathrm{R}_{\mathrm{Tt}} \mathrm{e}$ dada pela inclinação das retas da Figura 4:

$$
R a_{T t}=\frac{d T t}{d \log t a}
$$

Como pode ser constatado, a velocidade $\mathrm{Ra}_{\mathrm{Tt}}$ diminui linearmente em relação a $\mathrm{Tg}$-Ta. Isto indica que o processo de relaxação estrutural ainda está em evolução, isto é, o processo deverá continuar ocorrendo em sistemas curados por tempos maiores que $90 \mathrm{~min}$, a $115^{\circ} \mathrm{C}$.

A Figura 8 apresenta o módulo de armazenamento normalizado em função do logaritmo do tempo de envelhecimento (ta). Esta relação apresenta-se linear.

O módulo de armazenamento normalizado, $\log \mathrm{E}^{\prime} / \log \mathrm{E}^{\prime}{ }_{0}$ consiste no módulo de armazenamento, E' a $100^{\circ} \mathrm{C}$, em um tempo de envelhecimento, ta, dividido pelo módulo de armazenamento em ta $=0$ min, $E_{0}{ }_{0}$. Esta normalização visa atenuar ou, até, eliminar os efeitos associados com o processamento das amostras ${ }^{[17]}$.

A velocidade de envelhecimento a partir do módulo de armazenamento pode ser calculada a partir da inclinação das retas da Figura 8, segundo a equação ${ }^{[2,17]}$ :

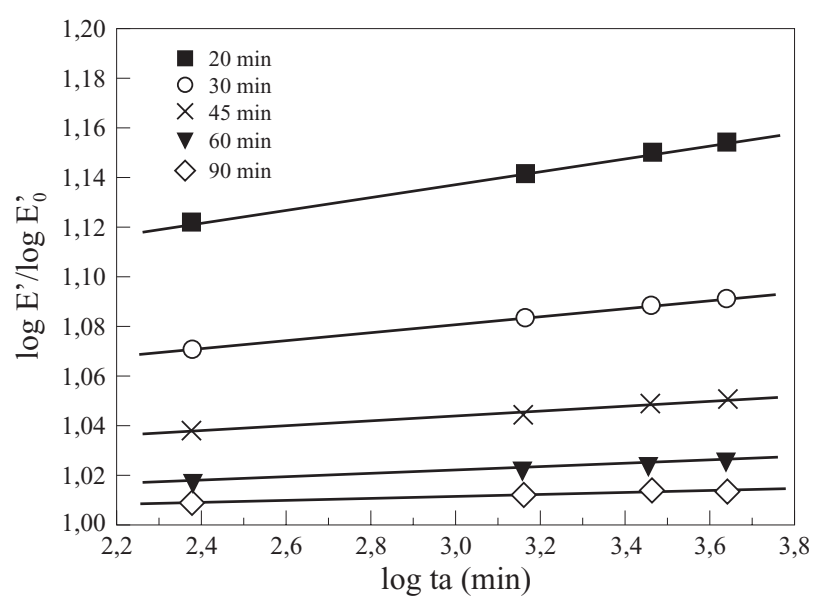

Figura 8. Módulo normalizado em função do tempo de envelhecimento. Tempos de cura indicados.

$$
R a_{E^{\prime}}=\frac{d\left(\log E^{\prime} / \log E_{0}^{\prime}\right)}{d(\log t a)}
$$

Na Figura 9 estão plotados os valores da velocidade $\mathrm{Ra}_{\mathrm{E}}$, em função de (Tg - Ta). Observa-se nesta figura que, quanto maior o afastamento entre a $\mathrm{Ta}$ e a $\mathrm{Tg}$, menor será $\mathrm{Ra}_{\mathrm{E}}$, segundo ajuste linear.

$\mathrm{O}$ mesmo procedimento anterior pode ser realizado utilizando-se os valores de Tg. A Tg normalizada consiste na $\mathrm{Tg}$ relativa a um tempo de envelhecimento ta, dividida por $\operatorname{Tg}_{0}$ que éa $\mathrm{Tg}$ da amostra a ser envelhecida, ta $=0$ min. A velocidade $\mathrm{Ra}_{\mathrm{Tg}}$ será, portanto:

$$
R a_{T g}=\frac{d\left(T g / T g_{0}\right)}{d(\log t a)}
$$

Também neste caso, $\mathrm{Ra}_{\mathrm{Tg}}$ e $\mathrm{Tg}$-Ta apresentam uma relação linear.

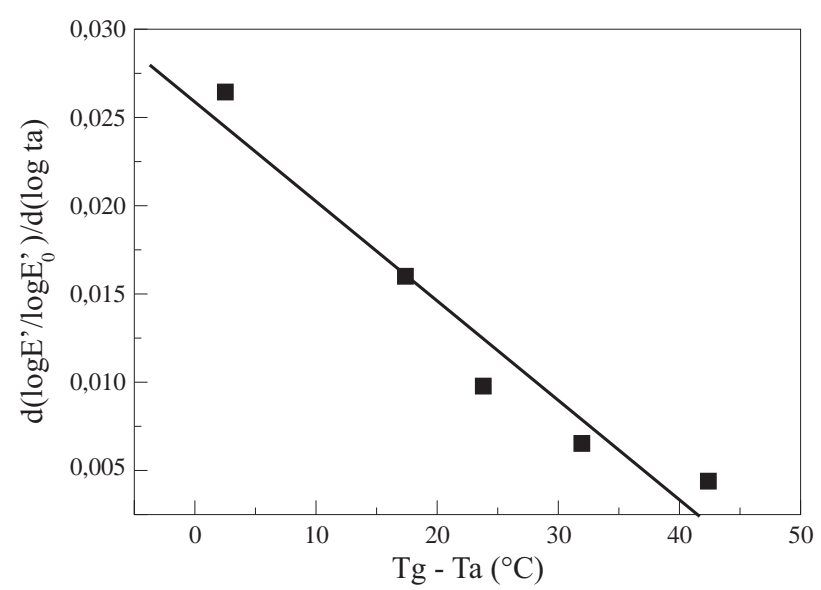

Figura 9. Velocidades de envelhecimento a partir do módulo de armazenamento normalizado em função de $(\mathrm{Tg}-\mathrm{Ta})$. 


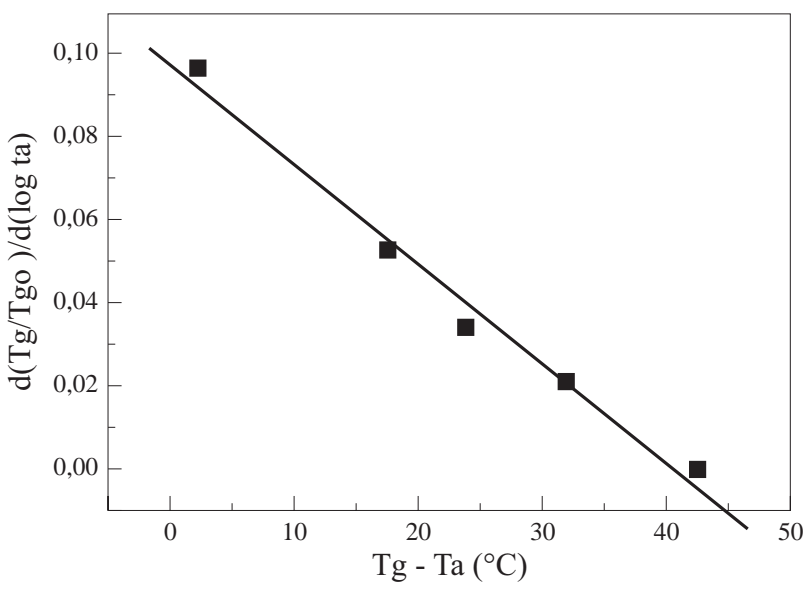

Figura 10. Velocidades de envelhecimento a partir da $\mathrm{Tg}$ normalizada em função de $(\mathrm{Tg}-\mathrm{Ta})$.

Comparando-se o comportamento das velocidades de envelhecimento definidas segundo as equações 3,4 e 5 , observa-se que todas relacionam-se linearmente com Tg-Ta. Portanto, tanto os valores de $\mathrm{Tt}$, de $\mathrm{E}^{\prime}{ }_{100}$ ou de $\mathrm{Tg}$ podem ser utilizados para o cálculo da velocidade de envelhecimento a $\mathrm{Ta}<\mathrm{Tg}$, pois ambos apresentam a mesma relação com TgTa, indicando o decréscimo linear da velocidade de envelhecimento a medida que aumenta o grau de conversão da matriz e conseqüentemente diminui a mobilidade da rede reticulada.

\section{Conclusões}

A entalpia de relaxação, que apresenta-se como um pico endotérmico nas curvas DSC das amostras envelhecidas, e a temperatura máxima de ocorrência (Tt) aumentam com o tempo de envelhecimento. Este efeito denota o avanço do processo de envelhecimento físico realizado, no qual maiores tempos de envelhecimento levam a relaxações caracterizadas por maiores variações de entalpia.

O módulo de armazenamento E' mostrou-se sensível às condições iniciais de cura dos sistemas epoxidílicos (conversão química) e também às alterações estruturais decorrentes do processo de envelhecimento. $\mathrm{O}$ valor do módulo $\mathrm{E}^{\prime}$ a $100^{\circ} \mathrm{C}, \mathrm{E}^{\prime}{ }_{100}$, apresenta uma tendência crescente devido ao processo de envelhecimento, para todos os sistemas estudados, evidenciando um aumento na rigidez do material.

A velocidade do envelhecimento físico definida através da temperatura do pico endotérmico, através do módulo de armazenamento ou através da temperatura de transição vítrea, decresce na medida que a Tg do sistema inicial aumenta, ou seja, quanto maior o grau de conversão, menor a velocidade, possivelmente devido à menor mobilidade molecular das estruturas mais densamente reticuladas.

\section{Referências Bibliográficas}

1. May, C.A. - "Epoxy Resins: Chemistry and Technology”, Marcel Dekker Inc., New York (1988).

2. Wang, X. \& Gillham, J.K. - J. Appl. Polym. Sci., 47, p.447 (1993).

3. Struik, L.C.E. - " Encyclopedia of Polymer Science and Engineering”, in: Aging,Physical, Vol 1, p.595, H. Mark, N. Bikales, C. Overberger \& G. Menges (ed.), John Wiley \& Sons, New York (1986).

4. Struik, L.C.E. - "Physical Aging in Amorphous Polymers and Other Materials", Elsevier, Amsterdam (1978).

5. McKenna, G.B. \& Lee, A. - Polymer, 29, p.1812 (1988).

6. McKenna, G.B. \& Lee, A. - Polymer, 31, p.423 (1990).

7. McKenna, G.B. \& G'Sell, C. - Polymer, 33, p.2103 (1992).

8. McKenna, G.B. \& Waldron, W.K. - J. Rheol., 39(2), p.471 (1995).

9. McKenna,G.B.; Leterrier, Y. \& Schultheisz, C.R. - Polym. Eng. Sci., 35, p.403 (1995).

10. Miranda, M.I.G. \& Samios, D. - Eur. Polym. J., 33:3, p.325 (1997).

11. Ophir, Z.H., Emerson, J. A \& Wilkes, G.L. - J. Appl. Polym. Sci, 49, p.5032 (1978).

12. Kreibich, U.T. \& Schmid, R. - J. Polym. Sci.; Symp., 53, p.177 (1975).

13. Montserrat, S. - J. Appl. Polym. Sci: Part B: Polym. Phys.,32, p.509 (1994).

14. Venditti, R.A. \& Gillham, J.K. - J. Appl. Polym. Sci., 45, p.1501 (1992).

15. Wang, X. \& Gillham, J.K. - J. Appl. Polym. Sci., 47, p.425 (1993).

16. Maddox, S.L. \& Gillham, J.K. - J. Appl. Polym. Sci., 64, p.55 (1997).

17. Brennan, A.B. \& Feller, F. - J. Rheol., 39(2), p.453 (1995).

Recebido: 13/04/99

Aprovado 27/09/99 